\title{
Maurice Lagueux
}

Professeur de philosophie, Université de Montréal

$$
2004
$$

\section{"Le marxisme est-il encore pertinent aujourd'hui ?"}

Un document produit en version numérique par Jean-Marie Tremblay, bénévole, Professeur sociologie au Cégep de Chicoutimi Courriel: jean-marie tremblay@uqac.ca

Dans le cadre de "Les classiques des sciences sociales"

Site web: http://classiques.uqac.ca/

Une bibliothèque fondée et dirigée par Jean-Marie Tremblay, sociologue

Une collection développée en collaboration avec la Bibliothèque

Paul-Émile-Boulet de l'Université du Québec à Chicoutimi

Site web: http://bibliotheque.uqac.ca/ 
Cette édition électronique a été réalisée par Jean-Marie Tremblay, bénévole, professeur de sociologie au Cégep de Chicoutimi à partir de :

Maurice Lagueux

Professeur de philosophie, Université de Montréal

\section{"Le marxisme est-il encore pertinent aujourd'hui ?"}

Un article publié dans la revue Recherches sociographiques, vol. XLV, no 2, mai-août 2004, pp. 289-305. Québec: Les Presses de l'Université Laval.

[Autorisation accordée le 23 novembre 2006 par l'auteur de diffuser cet article dans Les Classiques des sciences sociales.]

Courriel : maurice.lagueux@umontreal.ca

Site web : http://www.philo.umontreal.ca/dept/cahiers.html

Polices de caractères utilisée :

Pour le texte: Times New Roman, 14 points.

Pour les citations : Times New Roman 12 points.

Pour les notes de bas de page : Times New Roman, 12 points.

Édition électronique réalisée avec le traitement de textes Microsoft Word 2004 pour Macintosh.

Mise en page sur papier format

LETTRE (US letter), 8.5" x 11")

Édition numérique réalisée le 5 décembre 2006 à Chicoutimi, Ville de Saguenay, province de Québec, Canada. 
Maurice Lagueux

Professeur de philosophie, Université de Montréal

\section{"Le marxisme est-il encore pertinent aujourd'hui ?"}

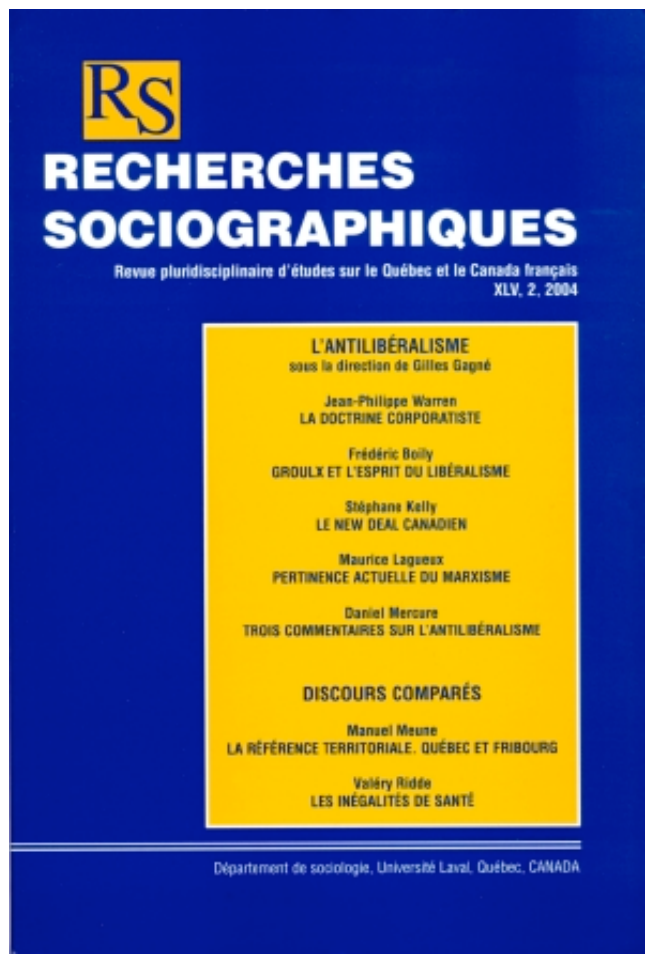

Un article publié dans la revue Recherches sociographiques, vol. XLV, no 2, mai-août 2004, pp. 289-305. Québec: Les Presses de l'Université Laval. 


\title{
Table des matières
}

\author{
Introduction
}

Peut-on appliquer la pensée de Marx?

Le marxisme comme instrument critique

Marxisme et néolibéralisme

Bibliographie 


\section{Maurice Lagueux \\ Professeur de philosophie, Université de Montréal \\ "Le marxisme est-il encore pertinent aujourd'hui ?"}

Un article publié dans la revue Recherches sociographiques, vol. XLV, no 2, mai-août 2004, pp. 289-305. Québec: Les Presses de l'Université Laval.

Quel rôle peut-on reconnaître au marxisme dans la critique du néolibéralisme? On aurait tort de lui refuser toute pertinence en invoquant le fait qu'au vu de ses échecs retentissants le marxisme ne peut offrir une solution de rechange valable. On aurait également tort d'y voir l'instrument théorique sur lequel devrait s'appuyer toute critique du néolibéralisme. Une analyse des évolutions respectives du marxisme et du néolibéralisme permet de conclure que c'est uniquement en tant que réalité sociale sui generis et non en tant que contribution théorique forcément dépassée que le marxisme se survit à lui-même dans une critique radicale du néolibéralisme.

\section{Introduction}

\section{$\underline{\text { Retour à la table des matières }}$}

Sans doute se demandera-t-on en quoi il peut être pertinent, au moment où il s'agit de s'interroger à propos des développements inquiétants du capitalisme néolibéral, de s'intéresser au marxisme, un courant de pensée qui, s'il a profondément marqué le milieu du XXe siècle, a désormais cessé d'exercer une influence politique significative dans la plupart des pays de la planète et de constituer, même pour les esprits les plus contestataires de notre époque, la référence fonda- 
mentale qu'il a jadis été ${ }^{1}$. Ces derniers, en effet, semblent plutôt rarement enclins à chercher leur inspiration dans les écrits de Marx quand ils s'en prennent au néolibéralisme qui, en ce début du XXIe siècle, paraît triompher et s'imposer partout. Et pour qui s'intéresse plus particulièrement à la situation propre au Québec, force est de reconnaître que le marxisme y a laissé bien peu de traces visibles de l'impact qu'il a pu y exercer. Nombreux sont ceux qui qualifieront même d'épiphénomène tout à fait superficiel l'engouement pour la pensée de Marx qui, l'espace d'à peine une décennie, s'est emparé d'une myriade de jeunes militants fraîchement convertis, lesquels, pour la plupart, devaient, au bout de quelques années, jeter à la poubelle leur petit livre rouge ou leur béret " à la Che » pour revendiquer au plus tôt la place enviable que leur promettait leur appartenance à la génération des baby-boomers.

Pourtant, on aurait bien tort de réduire à une sorte de rêve surréaliste un projet qui, pendant plusieurs années, a mobilisé à plein temps, ou presque, des centaines de jeunes et de moins jeunes, a fait trembler nombre de ceux qui détenaient l'autorité dans le monde académique, ecclésial, financier et même politique, a paralysé en maintes occasions de nombreuses institutions et a suscité de multiples commentaires et des enquêtes fort élaborées dans la plupart des médias du Québec. Bien qu'il soit périlleux de fixer des dates en vue d'encadrer un phénomène de ce genre, on peut voir dans la fondation de la revue Parti pris en 1963 le début de l'affirmation publique au Québec d'un mouvement qui allait se développer comme une traînée de poudre ou comme une épidémie - selon la métaphore qu'on jugera la plus appropriée en la circonstance - dans les milieux académiques et syndicaux. Dans la foulée de ce mouvement, même les intellectuels les moins en-

1 Une version abrégée de ce texte a été présentée sous le même titre, le 2 novembre 2001 au séminaire Fernand-Dumont consacré au thème « L'anti-libéralisme au Québec au XXe siècle ». Une autre version abrégée a été publiée sous le titre «Que reste-t-il du marxisme d'antan ? » dans Le Devoir des 17-18 novembre 2001. L'auteur remercie Francis Dupuy-Déry, son commentateur à ce séminaire, ainsi que Gilles Bibeau, Denys Delâge, Gilles Gagné, Daniel Jacques, Stéphane Kelly, Daniel Mercure, Jean-Marc Motte, Marc Saint-Laurent, Jean-Jacques Simard ainsi que les deux lecteurs anonymes de Recherches sociographiques de leurs remarques ou de leurs suggestions. Il remercie également le CRSH de son aide financière. 
gagés sentaient le besoin de « flirter » avec le marxisme, ne serait-ce que pour ne pas paraître dépassés aux yeux de leurs contemporains. Ceux, dont je suis, qui, au nom de convictions philosophiques, politiques, sociologiques, religieuses ou autres, refusaient de s'associer à ce mouvement pouvaient difficilement ne pas éprouver cette sorte de mauvaise conscience -je puis en témoigner personnellement - que provoque l'impression gênante de passer à côté de l'Histoire. Et pourtant le puissant attrait qu'exerçait la pensée de Marx au début des années 1970 a commencé à s'affadir dès la fin de cette décennie sans avoir pu laisser sa marque dans l'orientation politique effective du Québec, de sorte qu'avant même la chute des régimes communistes à la fin des années 1980, il n'était plus, au Québec, qu'un souvenir de moins en moins vivace.

Comme on peut néanmoins se demander si ce mouvement ne connaît pas une certaine résurgence depuis quelques années à la faveur des nombreuses levées de boucliers contre la mondialisation et contre les politiques néolibérales, il paraît pertinent de s'interroger sur ce qu'il fut vraiment et sur ce qui explique son étonnante évolution. je m'efforcerai de le faire, tout en tentant de répondre à la question de savoir si l'on doit s'attendre à ce que ce marxisme moribond renaisse de ses cendres en raison de l'effervescence nouvelle que connaît présentement la critique du capitalisme contemporain et de son orientation néolibérale.

D'un côté, on peut être enclin à répondre par l'affirmative à cette dernière question, en alléguant que le marxisme, même aujourd'hui, constitue un riche arsenal d'arguments dirigés contre le capitalisme libéral, arsenal dont les pièces sont dues tant à l'analyse scientifique que Marx a lui-même consacrée au capitalisme qu'aux contributions de ses innombrables disciples qui, depuis plus de cent ans, n'ont cessé de raffiner les objections qu'ils opposent à leurs adversaires libéraux. D'un autre côté, on peut penser que la réponse à la même question devrait d'emblée être négative, car une critique du capitalisme vaut ce que vaut la solution de remplacement proposée pour résoudre le problème posé par la mise en place d'une société à la fois juste et efficace ; or, sur ce terrain, le moins que l'on puisse dire, c'est que le marxisme ne peut faire mieux que le libéralisme, comme en font foi ses échecs lamentables - que ces échecs aient été consacrés par l'abandon 
ou par la trahison du projet marxien - dans tous les pays où l'on a essayé de l'appliquer.

\section{Peut-on appliquer la pensée de Marx ?}

\section{$\underline{\text { Retour à la table des matières }}$}

Or je pense que ces deux réponses ne sont pas satisfaisantes et je vais m'efforcer de le montrer en commençant par la deuxième. La pensée de Marx est-elle vraiment discréditée par les échecs rencontrés dans tous les pays où l'on a voulu l'appliquer? On peut, non sans raison, trouver bien injuste cette façon de voir puisque, tel que conçu par Marx, le communisme est littéralement aux antipodes du totalitarisme bureaucratique qui a été pratiqué dans la plupart des sociétés qualifiées de communistes. Dans les passages de son oeuvre où il fait part de sa conception d'une société communiste - que ce soit dans les Manuscrits de 1844 qu'il rédigeait en début de carrière, au moment même où il adoptait le communisme, ou dans les Closes dont, en 1875, vers la fin de sa carrière, il annotait le programme de Gotha -, Marx met invariablement l'accent sur le libre épanouissement des individus, sur la satisfaction des besoins humains les plus variés, sur la subordination de l'État à la société, etc. ${ }^{2}$. Qui plus est, aussi tôt qu'en 1843, il n'hésitait pas à formuler une critique aussi violente que lucide à l'endroit du type de bureaucratie dont les régimes communistes du XXe siècle devaient donner un si triste exemple ${ }^{3}$.

On aurait donc bien tort de voir dans les piètres résultats obtenus par les régimes communistes à travers le monde le fruit peu glorieux d'une application de la pensée de Marx, mais on aurait également tort de penser qu'il suffirait d'appliquer cette pensée plus correctement pour arriver à un résultat plus heureux, car le problème est que ce

2 MARX (1968, p. 84-99) et MARX (1963, pp. 1411-1434).

3 Critique de la philosophie politique de Hegel, dans MARX (1982, pp. 921922). 
communisme admirable dont Marx s'est fait l'éloquent propagandiste n'est rien d'autre qu'une utopie qui ne peut pas plus être " appliquée » que l'Utopie de Thomas More. Loin de moi l'idée de prendre la défense de Lénine et de Staline, mais il faut reconnaître que, quand ces soi-disant " représentants du peuple » se sont mis en frais de bâtir une société nouvelle, ils ne pouvaient que l'inventer de toutes pièces, car ils auraient difficilement pu trouver dans les écrits de Marx une réponse satisfaisante aux problèmes multiples que pose la mise en place d'une société communiste viable.

En particulier, Marx ne leur disait nulle part comment concilier participation et planification qui constituaient deux composantes essentielles mais apparemment incompatibles de ce communisme idéal. Or c'est à ce chapitre que la plupart de ces régimes ont échoué le plus lamentablement. Abolir le capitalisme n'allait pas sans supprimer les mécanismes décentralisés grâce auxquels les marchés libres assuraient une allocation des ressources relativement efficaces à bien des égards. Or, aux yeux des marxistes les plus convaincus, il n'y avait rien à regretter de ce point de vue, car l'allocation anonyme et brutale qu'exerçait le marché allait être remplacée par une façon beaucoup plus rationnelle en apparence de répartir les ressources puisqu'elle reposait non plus sur les aléas du marché mais sur une planification fondée sur une analyse des besoins essentiels de l'économie. Toute l'analyse de Marx était effectivement basée sur l'idée qu'il fallait substituer au mécanisme aveugle des marchés capitalistes une planification rationnelle des ressources, selon un mode que Marx n'a pas eu le temps d'analyser en détail mais dont l'efficacité était supposée par toutes ses descriptions de la société socialiste. Toutefois, qui dit planification dit décision. Mais qui doit prendre les décisions dans un régime socialiste engagé sur la voie de l'économie planifiée ? À cette question, le marxiste imbu des plus belles pages de Marx pouvait répondre sans hésiter que c'est au prolétariat lui-même qu'il revient de prendre de telles décisions, à ce prolétariat qui avait fait la révolution, qui avait renversé le capitalisme, qui s'était approprié les moyens de production, qui avait pris le pouvoir et qui exerçait même temporairement une " dictature » à laquelle devaient se soumettre les héritiers de la bourgeoisie.

Cette prise en charge du prolétariat par lui-même ne pouvait que susciter l'enthousiasme aux belles heures des flambées révolutionnai- 
res, mais quand fut venu le temps de prendre les vraies décisions, celles qui devaient forcément prendre appui sur de savantes analyses techniques ou sur la mise en balance de multiples intérêts, il a bien fallu se rendre compte qu'une classe sociale, aussi unifiée et aussi socialement éduquée qu'on puisse l'imaginer, peut difficilement s'exprimer a l'unisson. A vrai dire, elle le peut d'autant moins que ce n'est que métaphoriquement qu'on peut dire d'elle qu'elle s'exprime en tant que classe. Aux yeux de Lénine, il n'y avait pas là un véritable problème puisque le prolétariat allait pouvoir s'exprimer par l'intermédiaire du Parti qui, après tout, ne devait être rien d'autre que l'expression politique de sa volonté. Aussi, est-ce le Parti qui est devenu l'instance décisionnelle dont il fallait, en principe, respecter la volonté. Certes, un parti aussi solidement structuré que l'était le Parti communiste de l'Union soviétique était-il beaucoup plus en mesure que le prolétariat de prendre les décisions qui s'imposaient. Toutefois, il l'était précisément parce que le caractère hiérarchique de sa structure pouvait assurer efficacement une délégation de pouvoir entre les mains de quelques dirigeants, voire d'un seul potentat, en qui le Parti, et indirectement le prolétariat, étaient invités à reconnaître fictivement la voix chargée d'exprimer leurs volontés les plus profondes. Or, cette concentration du pouvoir, qui - on le sait aujourd'hui - n'a pas suffi, loin de là, à assurer le succès de cette planification, ruinait, dès le départ, toute velléité de participation populaire, d'autant plus qu'elle supposait l'abolition des droits qui, dans les démocraties occidentales, permettent aux citoyens de participer, aussi minimalement et indirectement que ce soit, aux orientations politiques de leur société.

Sans doute, a-t-on compris assez vite que s'il est possible pour les bureaucrates et les chefs de parti de prendre des décisions sans consulter ni le prolétariat ni les membres du parti, il n'est guère possible de mettre efficacement en oeuvre un plan économique sans que ces instances non consultées participent en quelque façon à sa réalisation. Aussi a-t-on cherché à s'assurer de cette participation à l'aide soit de ce que l'on a appelé des "stimulants matériels », soit de ce qui a été 
qualifié de "stimulants idéologiques ${ }^{4}$. Dans le premier cas, il s'agissait de mettre en œuvre des mécanismes vaguement modelés sur les marchés capitalistes, mais étroitement subordonnés aux diktats des planificateurs, de manière à ce que des primes viennent inciter les dirigeants d'entreprise et les travailleurs à réaliser les performances que le plan pouvait requérir. Dans le second cas, il s'agissait plutôt, à l'aide de slogans bien martelés et d'autres techniques de persuasion, de générer chez ces agents économiques, comme on l'a fait surtout en Chine, le sentiment de partager "spontanément» des décisions venues d'en haut mais entérinées dans l'enthousiasme par un peuple forcé de faire sienne la pensée du dirigeant suprême. Ces efforts pour souder ensemble participation et planification se sont manifestement soldés par des échecs. Ici encore, toutefois, on aurait tort d'en faire porter directement le blâme à Marx puisque les techniques employées, qu'il s'agisse de stimulants matériels ou de stimulants idéologiques, étaient parfaitement étrangères à sa pensée. Il n'en reste pas moins que si ces résultats ont été décevants à ce point, c'est que les dirigeants communistes qui ont imaginé ces techniques ne pouvaient trouver chez Marx de recettes plus efficaces pour harmoniser ces deux éléments essentiels d'une société politique fondée sur une application de ses idées.

Car, même si Marx n'a guère utilisé ce terme, la participation du prolétariat aux décisions qui devaient déterminer sa destinée était au cœur de son projet puisque ce prolétariat qui n'avait " rien d'autre à perdre que ses chaînes » devait s'approprier l'ensemble des moyens de production et devenir le détenteur ultime du pouvoir politique. Il semble donc que, malgré la perspicacité de ses analyses socio-économiques et malgré sa méfiance instinctive à l'égard des abstractions, Marx ne se soit guère demandé en vertu de quoi le prolétariat pourrait constituer une entité politique capable d'exprimer en tant que classe des volontés présumées communes à l'ensemble de ses membres. Sans doute ce qui semble tenir à une étonnante et inhabituelle naïveté de sa part s'explique-t-il, du moins en partie, par le fait qu'il était profondément convaincu que l'abolition de la propriété privée des moyens de

4 Sur ce sujet on pourra consulter l'ensemble d'un numéro consacré à la planification de Recherches internationales à la lumière du marxisme, no 47, maijuin 1965 ainsi que BETTELHEIM (1966), MANDEL (1965), ROBINSON (1969), MASSIP (1973) et LAGUEUX (1974, section III). 
production allait transformer radicalement les rapports entre les hommes. Aussi, lui paraissait-il probable que les prolétaires, jusque-là aliénés et divisés entre eux par les tensions que créaient les rapports de production capitalistes, sauraient, une fois « libérés de leurs chaînes », reconnaître leurs véritables intérêts et former un corps unifié capable de parler d'une seule voix. Sans doute ces prolétaires y sont-ils parvenus, mais pendant quelques moments de grâce seulement, au cours des heures enivrantes vécues à l'occasion de ces élans révolutionnaires dont la Commune de Paris offre sans doute le meilleur exemple.

Cette image utopique d'un prolétariat libre, à l'abri des conflits d'intérêts que des rapports de production basés sur la propriété privée suscitaient au sein des classes pré-révolutionnaires, est peut-être l'héritage le plus ambigu que Marx a légué aux générations de marxistes qui se sont succédé au cours du XXe siècle. On me permettra d'illustrer la chose à l'aide d'une expérience personnelle. Au début des années 1970, j'avais mis sur pied au département de philosophie de l'Université de Montréal un séminaire consacré à une réflexion philosophique sur l'écologie. Il était naturel, dans ce contexte, de souligner qu'une part importante de la pollution engendrée dans les pays capitalistes tient aux externalités que l'on observe, par exemple, quand des propriétaires d'usines se débarrassent de leurs déchets toxiques en les écoulant dans des rivières aux dépens des concitoyens qui habitent en aval, et ce, sans qu'interviennent de compensations financières du type de celles que les marchés assurent en d'autres circonstances. Certains étudiants en concluaient aussitôt que le communisme constituait une solution radicale à ces problèmes écologiques puisque, dans les sociétés communistes comme celles qui s'étaient implantées en URSS et en Europe de l'Est, les usines appartiennent au prolétariat ou, si l'on préfère, aux citoyens qui sont précisément ceux qui ont tout intérêt à ce que les territoires dont ils sont également les propriétaires collectifs ne soient pas pollués en aval. Qui serait assez stupide pour rendre invivable son propre domaine à force d'y déverser inconsidérément ses déchets ? Dans ce contexte, je ne pouvais parvenir à faire comprendre à ces étudiants que ces citoyens, dont l'intérêt premier était d'accroitre le plus possible la productivité de l'usine dont ils avaient la direction afin de se tailler une place enviable dans l'appareil du Parti, et ces autres citoyens, dont l'intérêt était de se baigner dans une eau propre en aval 
de cette usine, n'avaient pas forcément les mêmes intérêts, nonobstant le fait qu'ils étaient tous citoyens d'un pays où les moyens de production étaient par hypothèse de propriété publique. Ces considérations théoriques ne pesaient pas lourd face à la représentation enivrante d'une classe dont tous les membres partagent fondamentalement les mêmes intérêts parce que nul d'entre eux ne possède privément de moyens de production. Il est vrai qu'on ne savait pas à l'époque que les régimes qui se réclamaient de Marx étaient en train de polluer leurs territoires à un rythme rarement égalé dans les pays capitalistes avancés. L'image, véhiculée dans trop de textes de Marx, d'un prolétariat capable de prendre des décisions en fonction de "ses " intérêts propres aussi aisément que peut le faire un individu ne permettait tout simplement pas d'imaginer qu'une telle incurie puisse être possible ${ }^{5}$.

Bref, la société dont Marx s'est fait le promoteur est une société idyllique où chacun serait appelé à s'adonner à la chasse, à la pêche ou à la critique philosophique selon son bon plaisir ${ }^{6}$. Une telle société se situe nettement aux antipodes de ces sociétés communistes dont le XXe siècle nous a offert tant d'exemples avec lesquels il serait bien injuste de la confondre. Mais comme toutes les sociétés idylliques, cette attrayante société devait demeurer une utopie et c'est bien à tort qu'on a cru que, pour être à même de la mettre en place de façon viable, il suffisait d'effectuer une révolution politique. On ne saurait d'ailleurs s'en étonner quand on tient compte du fait que, au sein de son œuvre colossale, Marx n'a dédié à l'analyse du communisme qu'assez peu de pages - d'ailleurs beaucoup plus percutantes d'un point de vue rhétorique qu'originales d'un point de vue scientifique - puisque c'est à la critique du capitalisme qu'il a consacré la presque totalité de son oeuvre scientifique ${ }^{7}$.

5 Voir à ce propos KOMAROV (1981) et les chapitres 7 à 15 de PELT (1990).

6 Allusion au célèbre passage de l'Idéologie allemande, MARX (1982, p. 1065).

7 Pour une discussion des aspects positifs et négatifs de la pensée de Marx, voir LAGUEUX (1983). 


\section{Le marxisme comme instrument critique}

$\underline{\text { Retour à la table des matières }}$

Si le mérite scientifique de Marx tient à sa critique du capitalisme plutôt qu'à ses projets de société nouvelle, il est temps de revenir à la première réponse à ma question initiale, celle qui mettait l'accent sur l'efficacité de la pensée de Marx en tant que critique du capitalisme et du libéralisme. Il est toutefois douteux que cette réponse soit vraiment plus satisfaisante que celle que l'on vient d'examiner. Elle ne l'est pas dans la mesure où, même si des penseurs comme Louis Althusser et ses nombreux disciples ont martelé a qui mieux mieux, pendant des années, l'idée voulant que seule la " science marxiste » pouvait nous servir de guide en matière de critique sociale, on voit mal en quel sens on devrait encore à l'aube du XXIe siècle s'en remettre sans plus à l'analyse scientifique d'un penseur du XIXe siècle, si perspicace qu'il ait pu être à son époque. Après tout, les contributions proprement scientifiques de Marx, qui témoignaient certes d'une incontestable puissance analytique, ont fait l'objet - comme celles de beaucoup de ses contemporains d'ailleurs - de critiques assez dévastatrices qu'on ne peut indéfiniment feindre d'ignorer.

Les marxistes qui ont eu la lucidité de le reconnaître sans renoncer pour autant à se dire marxistes ont cherché systématiquement à réinterpréter ce qui, dans cet héritage, ne pouvait être sauvé qu'à ce prix. À la théorie de la valeur-travail, on a substitué une discussion métaphysique de la valeur et du travail abstrait ; à la loi de la baisse tendancielle du taux de profit, on a substitué une analyse circonstanciée de l'évolution des profits, tantôt à la hausse, tantôt à la baisse ; à l'analyse du processus de décomposition du capitalisme, on a substitué une analyse des imprévisibles mutations de ce capitalisme; à la théorie de la lutte des classes, on a substitué une analyse sociologique des subtils modes de domination de la classe dominante, analyse plus fine certes, mais aux conclusions beaucoup moins percutantes. Bref, le marxisme a survécu au prix de mutations radicales de ce qui constituait la pensée de Marx au point où ce que l'on désigne encore du nom de marxisme ne repose plus guère sur les thèses brillantes mais contestables qui ont 
fait la fortune intellectuelle de Karl Marx. Peut-être, fera-t-on observer que, aujourd'hui encore, comme à toutes les époques de son histoire, le marxisme peut néanmoins se reconnaître à des traits comme la condamnation radicale de la propriété privée des moyens de production et la promotion inconditionnelle d'une société communiste. Il est incontestable que les marxistes pourraient difficilement renoncer à ces deux composantes de la pensée marxienne, mais il s'agit là de traits qui ne sont pas propres à Marx qui, s'il s'était contenté de se faire le dénonciateur de la propriété privée et le promoteur d'une société communiste, serait sans doute demeuré un obscur scribouilleur que l'Histoire n'aurait guère su distinguer des autres apôtres du socialisme qui, au XIXe siècle, sympathisaient avec de telles idées. Si Marx fut un penseur profondément original destiné à exercer une influence exceptionnelle sur la vie et la pensée politique au XXe siècle, c'est qu'il a su insérer ces considérations relativement banales dans une analyse socio-économique qui, à la fin du XIXe siècle, méritait largement, malgré la faiblesse de son versant proprement politique, d'être considérée comme la première analyse scientifiquement convaincante des phénomènes sociaux. Or ce sont ces conclusions scientifiques, qui ont fait la renommée de Marx, que les marxistes du XXe siècle ont peu à peu remplacées par des idées mieux adaptées aux besoins de leur époque mais relativement étrangères à la pensée du maître. Il est vrai que ce marxisme mille fois reformulé par des générations de penseurs de toute appartenance académique, au point de ne rien retenir d'autre de la pensée de Marx que sa volonté de substituer au capitalisme un mode de production jugé acceptable, a donné lieu à nombre de travaux de qualité souvent indéniable. Toutefois, la première question que l'on doit se poser à propos de ce marxisme est celle qui a trait à son étrange statut.

De quoi s'agit-il, en effet, quand on parle du marxisme ? Puisqu'il fait bon accueil aux réinterprétations les plus disparates, le marxisme pourrait difficilement être défini par la fidélité à la pensée de Marx, au sens ou l'hégélianisme, par exemple, peut être défini par la fidélité à la pensée de Hegel. C'est pourquoi le guerillero qui, sans rien connaître de l'oeuvre de Marx, s'attaque au péril de sa vie aux institutions capitalistes dans l'espoir de les renverser a-t-il cent fois raison de se déclarer authentiquement marxiste, alors qu'on imagine mal qu'un individu ignorant tout de l'œuvre de Hegel puisse être qualifié d'authentique 
hégélien! Sans doute, les praticiens du marxisme de cette trempe, engagés dans une lutte sans merci contre le capitalisme, savent-ils recycler en puissants slogans révolutionnaires nombre de notions auxquelles la théorie de Marx faisait appel : exploitation de l'homme par l'homme, concurrence sauvage, accumulation de plus-value, armée de réserve des travailleurs, lutte de classes, crises et contradictions du capitalisme, dictature du prolétariat, etc. Toutefois, ce qui fondamentalement les rattache au marxisme n'est plus une pensée bien structurée comme l'était celle de Marx, car cette structure est définitivement défaite et très souvent inconnue d'eux ; c'est bien plutôt un mouvement au sens sociologique du terme, soit celui qui, depuis plus d'un siècle déjà, consacre une sorte de parenté idéologique entre tous ceux et celles qui entretiennent l'idée qu'il est essentiel de renverser le capitalisme pour lui substituer une société que l'on voudrait plus humaine.

Mais comment le marxisme a-t-il pu être identifié ainsi à un mouvement qui déborde si largement la pensée de Marx ? Qu'est-ce qui a pu lui valoir ce statut privilégié ? Il semble bien que ce soit le fait que les écrits de Marx, pour la première fois dans l'histoire de l'humanité, ont su offrir à des générations d'individus assoiffés de justice et de vérité des raisons sérieuses de se convaincre que la promotion de la justice sociale et celle de la vérité scientifique ne constituaient qu'une seule et même cause. Bien des êtres généreux ont, de tout temps, voué leur vie à faire triompher la justice et la fraternité, mais jamais - du moins avant Marx - leur action pouvait-elle de façon crédible se réclamer de la pensée scientifique. Par ailleurs, bien des savants n'ont vécu que pour la science et ont mérité, à ce titre, la vénération de leurs congénères, mais, même si certains d'entre eux entretenaient de louables préoccupations sociales, leur réussite scientifique ne contribuait pas vraiment à l'avancement des causes sociales qu'ils auraient pu valoriser. Par contre, à la fin du XIXe siècle et même durant une bonne partie du XXe, les marxistes avaient des raisons tout à fait respectables d'estimer que la lutte pour le triomphe d'une société juste et fraternelle dans laquelle Marx les incitait à s'engager ne faisait qu'un avec la promotion de la seule analyse scientifique de la société et de l'histoire vraiment valable.

C'était plus qu'il n'en fallait pour cimenter cette mouvance sociale inédite - vite consolidée par les succès apparents de tant de révolu- 
tions qui se réclamaient de Marx - et pour conférer ainsi au marxisme son statut très particulier. Si la pensée de Marx n'a joué, comme on l'a vu, qu'un rôle bien limité dans la mise en place des structures sociopolitiques qui ont caractérisé les régimes socialistes du XXe siècle, elle a, par contre, joué un rôle crucial dans la radicalisation du mouvement révolutionnaire qui a permis à ces derniers de prendre forme. Comment, en effet, autant d'êtres généreux, qui n'avaient rien de fanatiques religieux, auraient-ils pu se résoudre à tuer tant de concitoyens au nom de la fraternité universelle s'ils n'avaient été convaincus, par une analyse que seul son caractère «scientifique » rendait à ce point crédible, qu'une telle action était requise pour que l'Humanité puisse enfin s'émanciper ? Une fois convaincus que Marx a scientifiquement établi dans le Capital que l'histoire exige le renversement du capitalisme et que seule cette étape cruciale peut permettre la mise en place d'une société où serait enfin abolie l'exploitation de l'homme par l'homme, ces êtres généreux pouvaient se résoudre à s'engager dans une action révolutionnaire, au besoin violente, pour faire advenir le plus tôt possible la révolution inévitable et surtout une société fondée sur des valeurs susceptibles de répondre aux véritables aspirations de l'humanité. Or, pour ces admirateurs de Marx, le Capital était à la science des sociétés ce que les Principia mathematica de Newton étaient à la science physique. Parce que Marx a été l'un des tout premiers à analyser les questions sociales à l'aide de méthodes qui, à l'époque, pouvaient être considérées comme rigoureusement scientifiques, sa pensée a pu être perçue par ceux qu'elle rejoignait comme la première et la seule véritable analyse scientifique des phénomènes sociaux. C'en était assez pour qu'elle exerce le rôle idéologique qu'elle a effectivement exercé en apportant aux révolutionnaires du XXe siècle la puissante caution morale dont ils avaient grandement besoin pour poursuivre leurs audacieuses et éprouvantes entreprises. Seule une théorie hautement crédible peut offrir un support idéologique efficace à ceux qui recherchent les justifications nécessaires pour affermir leur volonté de s'engager dans une action qui semble si souvent contre-productive. Or, dans le monde moderne, rien n'est plus crédible que la science. Aussi sont-ce le plus souvent d'authentiques contributions scientifiques qui, dans ce monde moderne, ont été mises avec succès au service des idéologies les plus diverses. C'est pourquoi la pensée de Marx n'aurait jamais pu exercer l'immense influence idéologique qui 
fut la sienne si on n'avait pas eu de raisons sérieuses d'y voir une contribution scientifique hautement respectable ${ }^{8}$.

Si les jeunes universitaires marxistes qui ont fondé Parti pris étaient d'abord des poètes et des philosophes imbus des analyses de Jean-Paul Sartre et de Franz Fanon dont ils s'efforçaient avec enthousiasme et éloquence d'adapter les idées à la situation québécoise, ceux qui leur ont succédé ont cherché davantage à tirer parti des analyses du Capital de Marx, encouragés qu'ils étaient par l'insistance et la conviction avec laquelle Althusser et ses disciples répétaient à satiété que Marx était le fondateur de la science historique, en ajoutant même qu'il ne pouvait guère y avoir de science du social en dehors de ce qui était alors très sérieusement désigné du nom de "science marxiste ». Même si elle était, le plus souvent, acquise par procuration, la conviction que l'œuvre de Marx reposait sur des bases scientifiques inébranlables a sans doute joué un rôle déterminant dans l'engagement inconditionnel de tant de jeunes militants qui ont choisi de se tourner vers une action plus structurée au sein d'organisations ou de groupuscules d'allégeance trotskyste ou maoïste. Eux qui n'avaient même pas le bénéfice de se sentir portés par la mouvance inspiratrice d'une vraie révolution, comment auraient-ils pu accepter de sacrifier leur jeunesse en se laissant embrigader dans un militantisme aussi rigide qu'exigeant s'ils n'avaient pas été convaincus du fondement scientifique des mots d'ordre auxquels ils acceptaient d'obéir aussi aveuglément ? Cela ne veut pas dire qu'ils étaient tous en mesure d'évaluer la portée scientifique des thèses de Marx ni même qu'ils se préoccupaient vraiment de le faire ; mais combien parmi ceux qui vouent un culte inconditionnel a la science contemporaine sont en mesure d'évaluer la portée scientifique de la biologie moléculaire ou de la physique quantique ? Pour ceux et celles qui s'engageaient ainsi dans l'action marxiste, la science n'était rien d'autre que la seule connaissance vraiment fiable parce qu'ils pouvaient y voir une connaissance éprouvée à maintes reprises et soumise aux exigences de la raison, de cette raison qui a permis de se libérer des superstitions et des croyances du passé, qui a permis de comprendre le mouvement des astres et l'évolution de la vie, qui a permis d'arraisonner le monde matériel et qui, maintenant,

8 Voir à ce sujet, la section intitulée « Qu'est-ce qu'une idéologie ? » dans LAGUEUX (1995). 
grâce aux principes mis en lumière par Marx, allait permettre de construire une société sans classes et donc sans conflits sociaux.

Aussi, quand est venue l'heure des grands désenchantements, que le miracle chinois est apparu tellement moins enthousiasmant qu'il n'avait d'abord semblé, que les mystifications du discours althussérien furent reconnues pour ce qu'elles étaient ${ }^{9}$, que la " science marxiste " s'est révélée parfaitement impuissante à réaliser les rêves les plus légitimes, plusieurs parmi ces marxistes déçus n'ont eu d'autres choix que de se recycler. Ils avaient sincèrement consacré toute leur énergie à travailler à la construction d'une société plus juste et plus fraternelle et ils l'avaient fait avec la conviction que leur action ne pouvait qu'accompagner le développement de la pensée scientifique qu'ils espéraient par-dessus tout voir triompher des erreurs et des superstitions qui paralysent encore le développement normal des sociétés. Or ce dont ils se voyaient désormais privés, c'était de la conviction - jadis offerte par cette « science marxiste » qui leur glissait maintenant entre les doigts - que les combats pour la justice sociale ne faisaient qu'un avec la promotion de la pensée scientifique. Aussi, fidèles à leur premier engagement, plusieurs d'entre eux ont-ils continué, les uns à défendre dans le cadre d'un militantisme plus conventionnel les causes qu'ils estimaient justes, et les autres à mettre leur intérêt pour la science et pour la recherche au service de la poursuite d'une carrière scientifique dans le monde académique.

C'est dans ce contexte issu d'une étonnante évolution des choses qu'il faut se demander si le marxisme peut encore contribuer efficacement à la critique du capitalisme libéral. Il paraît assez clair que la pensée de Marx en tant que telle ne peut plus se donner comme critique appropriée du capitalisme, d'autant que le capitalisme contemporain est fort différent de celui que Marx prenait pour cible. Il parait encore plus évident, on l'a rappelé, qu'elle ne peut, de façon crédible, opposer une solution de rechange au capitalisme. Dans ce contexte, on ne voit guère comment la pensée de Marx pourrait être réactualisée de façon convaincante. Mais le marxisme conçu comme le mouvement social que je viens de décrire n'a pas à être réactualisé. Au cours des heures de gloire qu'il a connues au XXe siècle, le marxisme n'a jamais

9 À ce propos voir LAGUEUX (1982, p. 77-95). 
été très fidèle à la pensée de Marx, sans que cela n'incite tellement ses plus illustres représentants à chercher à le réactualiser sous sa forme la plus authentique. Ils n'avaient pas à le faire car, à cause du rôle idéologique exceptionnel que la pensée de Marx a joué auprès de tous les mouvements révolutionnaires du XXe siècle, le " marxisme » est devenu, par définition, en quelque sorte, le nom que l'histoire a donné à toute critique radicale du capitalisme, pour peu que, dans le cadre de la mouvance dont il a été question, elle se reconnaisse une filiation, aussi vague soit-elle, à l'égard de la pensée de Marx. Bref, ce marxisme qui est devenu une réalité sociale plutôt que théorique, est d'autant plus indéracinable que sa destinée est désormais à peu près totalement indépendante de celle de la pensée de son fondateur.

\section{Marxisme et néolibéralisme}

$\underline{\text { Retour à la table des matières }}$

Pour bien voir comment cette mouvance marxiste peut rejoindre la critique actuelle du capitalisme néolibéral, il faut d'abord se demander ce qui caractérise ce néolibéralisme qui semble colorer si intensément le capitalisme contemporain. On ne peut caractériser le néolibéralisme sans définir d'abord le libéralisme que le néolibéralisme entend, en quelque sorte, réinterpréter. Or le libéralisme, on le sait, est une notion particulièrement ambiguë. À l'origine, ce terme désigne un courant de pensée auquel on voit mal comment le marxisme aurait pu s'opposer. Tout comme le marxisme, le libéralisme s'est abreuvé aux idées qui ont caractérisé l'époque des Lumières et les débuts de la modernité. L'affirmation des droits de l'homme et des libertés fondamentales ainsi que la contestation de toute autorité jugée arbitraire - tant celle du Prince que celle qu'on associait aux coutumes fondées sur des traditions bien établies - sont à l'origine du libéralisme du XVIIIe siècle. Or, il s'agissait là de positions que Marx avait fait siennes avant même de découvrir le communisme et auxquelles il demeura viscéralement fidèle durant toute son existence.

Pour les libéraux qui, aux États-Unis, avaient rejeté l'autorité britannique et bâti de toutes pièces un État moderne, le libéralisme sug- 
gérait d'utiliser cet instrument étatique pour veiller à assurer à la fois la liberté individuelle de chaque citoyen et ce degré d'égalité et donc de justice que l'autorité arbitraire des puissants de la Métropole avait rendu inaccessible. On comprend donc que, dans ce contexte, ce sont ceux qui ont voulu recourir à l'État pour défendre cet idéal qui furent depuis lors qualifiés de " libéraux ». En Europe, par contre, les libéraux, estimant qu'il valait mieux « laisser faire » les individus que de leur imposer des décisions forcément arbitraires, n'ont pas eu le loisir de reconstruire de toutes pièces un État façonné à partir de leurs conceptions, de sorte qu'ils ont dû continuer de lutter pour la cause de la liberté contre les divers régimes qui se sont succédé sans répondre à leurs aspirations. Dans ce contexte, les luttes pour la liberté et pour la justice égalitaire pouvaient difficilement être menées de front, de sorte que ces libéraux n'ont pas tardé à se heurter aux socialistes pour qui le rejet des anciens régimes devait ouvrir la voie à une société plus juste, fût-ce aux dépens de la liberté individuelle qu'il était préférable selon eux de subordonner aux interventions d'une autorité plus éclairée. Cette tension, d'ailleurs présente avant même l'avènement de l'idéologie socialiste et jamais résolue par la suite, a forcé certains libéraux notoires - tel Adam Smith qui n'hésitait pas à reconnaître d'importantes responsabilités à l'État - à se montrer plus prudents dans leur défense de la liberté individuelle. Quant aux économistes qui, au cours des deux siècles qui ont suivi la publication de Wealth of Nations, ont poursuivi - tels John Stuart Mill, Léon Walras, John Maynard Keynes et Paul Samuelson - l'analyse des vertus du marché libre, ils ont de plus en plus insisté sur la nécessité d'une intervention étatique, ne serait-ce que pour mettre en place les conditions très restrictives qui, à leurs yeux, permettraient seules à ce marché libre de fonctionner de manière à la fois efficace et moralement acceptable. Au cours des années 1930, alors que le capitalisme semblait en voie de rendre l'âme dans les affres de la grande crise économique et que le socialisme soviétique semblait en mesure, grâce à ses plans quinquennaux, de faire prévaloir la raison sur le chaos économique, le libéralisme ne semblait plus avoir beaucoup d'avenir. Déjà, même chez ceux qui redoutaient une telle éventualité, des voix se faisaient entendre pour lesquelles la victoire définitive du socialisme n'était plus qu'une affaire de temps.

Certes, le capitalisme allait-il retrouver toute sa vigueur et même au cours des années, qualifiées par la suite de «trente glorieuses », qui 
se sont achevées vers le début de la décennie 1970 - connaître la plus impressionnante période de croissance de son histoire ; mais ce devait être au prix d'une intervention massive de l'État. Or, la croissance de l'État, de la fiscalité et de la dette nationale, associée à cette intervention accrue, a vite fait d'apparaître comme un remède plus grave que le mal aux quelques irréductibles défenseurs du libéralisme dont l'inquiétude s'est progressivement propagée auprès de couches de plus en plus importantes de la population. Cette présence croissante de l'État, jugée beaucoup trop massive et explicitement perçue comme le symptôme d'un glissement vers le socialisme, suscitait d'autant plus d'hostilité au cours des années 1970 et surtout des années 1980 qu'il devenait évident que les régimes socialistes se heurtaient, pour leur part, à des problèmes de fonctionnement de plus en plus difficiles à masquer. Aussi, quand le communisme s'est littéralement effondré à la fin des années 1980, le néolibéralisme qui, en Occident, avait déjà fort bonne presse dans les cabinets ministériels et dans plusieurs départements universitaires s'est-il vu octroyer une crédibilité inespérée auprès de la population en général aux yeux de laquelle l'échec du communisme paraissait confirmer de façon spectaculaire le bien-fondé d'une doctrine qui en constituait l'antithèse.

Ce néolibéralisme était un retour aux intuitions qui avaient conduit les libéraux du XVIIIe siècle à célébrer les vertus de la liberté, à ceci près que, face aux déboires rencontrés par les politiques interventionnistes et surtout par le socialisme, cette position s'y affirmait de façon beaucoup plus radicale. D'une part, puisque l'interventionnisme et le socialisme n'ont guère réussi à résoudre de façon satisfaisante les injustices sociales qu'ils dénonçaient, on avait moins de mal à soutenir que le libre fonctionnement des marchés, complété par une intervention minimale de l'État, saurait mieux remédier à celles-ci, tout en permettant d'accroître plus efficacement la richesse générale. D'autre part, les défenseurs d'un libéralisme ainsi renouvelé pouvaient appuyer certaines de leurs conclusions sur des analyses scientifiques qui leur faisaient défaut au cours de la longue période pendant laquelle le libéralisme avait progressivement perdu sa crédibilité au profit de l'interventionnisme et du socialisme. Aux mains des économistes de l'Université de Chicago et de ceux qui se sont engagés dans leur sillage, le néolibéralisme a inspiré d'importantes contributions à la science économique. Toutefois, ce succès même a vite été à son tour 
exploité idéologiquement pour justifier les comportements agressifs de capitalistes qui avaient désormais les mains libres, tout comme leur allié naturel, le gouvernement américain, qui ne rencontrait plus guère de résistance dans sa volonté d'étendre ses tentacules dans toutes les régions du monde. Tout comme le caractère scientifique - au sens qui s'applique aux analyses économiques du dernier tiers du XIXe siècle de certaines contributions de Marx a pu être mis au service de la volonté révolutionnaire des adversaires du capitalisme, le caractère scientifique - au sens qui s'applique aux analyses économiques du dernier tiers du XXe siècle - de diverses contributions des économistes d'orientation néolibérale a pu être mis au service des ambitions illimitées des grands capitalistes contemporains.

C'est à cet état de choses que font face aujourd'hui ceux qui s'engagent dans des manifestations qui prennent pour cible non seulement le capitalisme, mais ce néolibéralisme qui l'inspire ainsi que le phénomène même de mondialisation à la faveur duquel le capitalisme parvient à étendre son emprise. Étant donné la multiplicité des causes qu'elles entendent promouvoir, ces manifestations - où les revendications anarchistes, écologistes, féministes, et autres se joignent aux contestations radicales du capitalisme néolibéral et des politiques adoptées par les pays les plus puissants - sont assez différentes de celles que mettaient en branle les Marxistes de jadis. Il est douteux, on l'a $\mathrm{vu}$, que les thèses de Marx puissent être d'un grand secours à ceux qui s'engagent dans ces contestations, car les structures socio-économiques sur lesquelles s'appuie le capitalisme à l'aube du XXIe siècle diffèrent trop de celles qui caractérisaient la fin du XIXe siècle pour qu'une analyse, si perspicace soit-elle, de ces dernières puisse s'appliquer utilement aux premières. Et vu le peu de crédibilité scientifique dont jouit présentement la pensée de Marx auprès de la vaste majorité des intellectuels, il est aussi très peu probable que le marxisme puisse apporter aux contestataires d'aujourd'hui une caution idéologique du type de celle qu'il a su si efficacement offrir aux révolutionnaires de jadis, tant il est vrai que seule une théorie suffisamment crédible peut exercer une action idéologique efficace.

Au Québec, où la volonté d'abattre le système capitaliste ne se fait pas particulièrement insistante à notre époque, il est peu probable que le marxisme retrouve, même auprès de ceux qui sont inquiétés et indi- 
gnés par les orientations néolibérales de nos gouvernants, l'emprise qu'il a jadis exercée sur les esprits contestataires. Toutefois, on l'a vu, il ne faut pas confondre le marxisme et la pensée de Marx en tant que telle ; or, au Québec, cette dernière aura connu un destin qui, avec un décalage d'une génération, s'est révélé assez similaire à celui qui fut réservé à la pensée de Thomas d'Aquin. Au début des années 1960, le Docteur angélique dont l'Église catholique avait longtemps cherché à imposer la pensée dans toutes les sphères de l'activité humaine, a vu brusquement son influence réduite presque à néant auprès de générations qui en avaient assez d'entendre, à propos des moindres questions, marteler des solutions qui émanaient inlassablement d'une pensée unique. Aujourd'hui encore, le nom de saint Thomas éveille un souvenir plutôt amer chez nombre de ceux qui ont connu l'enseignement thomiste des collèges classiques, que le dogmatisme et le prosélytisme de tant de ses dispensateurs avaient rendu littéralement insupportable. Pourtant, dès les années 1980, plusieurs jeunes, qui n'avaient rien connu de cette époque, redécouvraient avec beaucoup d'intérêt l'éminent penseur du Moyen Âge qui a su aborder avec tant d'ampleur de vue les problèmes les plus complexes qui se posaient en son temps. De même, au cours de ces années 1980, le père du « socialisme scientifique », dont la pensée avait envahi l'enseignement de diverses disciplines tant au cégep qu'à l'université, a vu brusquement son influence réduite presque à néant auprès de ceux qui en avaient assez d'entendre répéter à tout propos par les chantres du matérialisme dialectique qu'il fallait renverser le système capitaliste et assurer ainsi la victoire du prolétariat, au sein duquel, solidarité oblige, les « travailleurs intellectuels »devaient trouver la place qui leur était dévolue. Ceux qui ont connu cette époque, où régnait dans bien des milieux une pensée unique, en gardent généralement un souvenir assez amer et ils ne sont guère enclins à se tourner à nouveau vers la pensée de Marx qui, dans leur esprit, est forcément associée à des formules qui, dans le contexte où elles étaient proférées, rendaient un son assez creux. Toutefois, depuis quelques années, des jeunes, dont plusieurs n'étaient pas nés au moment où le marxisme sévissait dans les institutions québécoises, redécouvrent avec beaucoup d'intérêt l'éminent penseur du XIXe siècle qui a su analyser, mieux que tous ses contemporains peut être, l'extraordinaire mutation de l'humanité qui en Occident a accompagné la Révolution industrielle. Marx, tout comme Thomas d'Aquin avant lui, redevenait l'un de ces grands « classiques » de l'humanité qu'il importe 
d'étudier attentivement pour mieux comprendre ce que nous sommes devenus, mais a qui nous ne saurions, sans profonde aberration, attribuer une " pensée » permettant de répondre systématiquement à toutes les questions posées par notre époque ${ }^{10}$.

Toutefois comme la pensée de Marx et le marxisme militant connaissent maintenant des destins largement indépendants, on peut se demander si le marxisme peut encore renaître de ses cendres. S'il y parvient, ce ne saurait être grâce à une réactualisation de la pensée de Marx qui peut certes intéresser les marxologues et tous ceux qui veulent mieux comprendre le devenir de notre civilisation, mais qui ne peut rendre compte de façon particulièrement utile des enjeux actuels. Si le marxisme peut, dans ce contexte, redevenir une force réelle, c'est plutôt en tant qu'il n'est rien d'autre que cette réalité sociale évoquée plus haut, cette mouvance révolutionnaire qui continue d'ailleurs d'exercer une influence non négligeable dans les pays moins développés où la lutte révolutionnaire de style traditionnel est toujours à l'ordre du jour. Cette volonté radicale de renverser le capitalisme dans l'espoir de lui substituer une société conforme aux aspirations humaines les plus fondamentales aura trop profondément marqué les comportements et les espoirs d'une multitude d'individus appartenant à des générations qui se sont succédé tout au long du XXe siècle pour s'estomper aussi facilement. Il serait étonnant que la culture marxiste qui s'est formée au cours de cette aventure historique qui n'a pas été menée à terme n'ait pas laissé de traces à peu près indélébiles ; aussi est-il à prévoir que cette culture marxiste refera surface partout où l'on conclura, pour quelque raison que ce soit, à la nécessité d'abolir le capitalisme. Sans doute, la pensée de Marx risquera-t-elle alors de n'être plus qu'un vague et lointain souvenir, mais ce sera le souvenir de cette idéologie qui a rendu possible la formation de cette culture révolutionnaire qui a animé pendant des décennies cette mouvance sociale qu'on a appelée « le marxisme ». C'est pourquoi on voit mal comment ceux qui s'en prennent à la mondialisation et au néolibéralisme échapperaient à l'emprise de cette mouvance marxiste, quand ils se radicali-

10 C'est en des termes assez voisins que, dès 1960, Maurice Merleau-Ponty, qui retrouvait alors une évidente sérénité après avoir péniblement abandonné ses convictions marxistes, expliquait que Marx était en train de devenir un « classique »(MERLEAU-PONTY, 1960, pp. 16-17). 
sent au point de conclure, comme les marxistes d'antan, que le capitalisme, dont les vices seraient accentués par le néolibéralisme, ne peut être amendé et qu'il faut donc le renverser. Aussi, quelle que soit l'efficacité actuelle de ce marxisme polymorphe, peut-on s'attendre à le voir reprendre du service tant que le capitalisme et les criantes inégalités qu'il engendre forcément dans un monde dominé par une philosophie néolibérale seront là pour lui servir de cible et de prétexte pour survivre.

Maurice LAGUEUX

Département de philosophie, Université de Montréal.

\section{BIBLIOGRAPHIE}

$\underline{\text { Retour à la table des matières }}$

BETTELHEIM, Charles, Problèmes théoriques et pratiques de la planification, 3e édition, Paris, Maspéro, 1966.

KOMAROV, Boris, Le rouge \& le vert. La destruction de la nature en URSS, Paris, Seuil, 1981. (Traduit du russe par Basile Karlinsky.)

\section{LAGUEUX, Maurice}

, « L'arrière-fond philosophique du concept de plan », Philosophiques, 1,1974 , pp. 51-82.

, Le marxisme des années soixante, Montréal, Hurtubise $\mathrm{HMH}, 1982$. [Texte disponible dans Les Classiques des sciences sociales. JMT.]

, "Grandeur et misère du socialisme scientifique », Philosophiques, no X, 1983, pp. 315-340.

, « Historiographie, philosophie de l'histoire et idéologie. À propos d'un texte de Fernand Dumont », dans : Simon LANGLOIS et Yves MARTIN (dirs), L'Horizon de la culture. Hommage à Fernand Dumont, Québec, Les Presses de l'Université Laval, 1995, pp. 95-108. [Texte disponible dans Les Classiques des sciences sociales. JMT.] 
MANDEL, Ernest, " La réforme de la planification soviétique et ses implications théoriques », Les temps Modernes, no 229, 1965, pp. 2164-2168.

\section{MARX, Karl}

, Oeuvres, tome I, Économie, traduction de Maximilien Rubel, Paris, Gallimard, 1963. (La Pléiade.) , Manuscrits de 1844, Paris, Éditions sociales, 1968. , Oeuvres, tome III, Philosophie, traduction de Maximilien Rubel et collaborateurs, Paris, Gallimard, 1982. (La Pléiade.)

MASSIP, Roger, La Chine est un miracle, Paris, Bernard Grasset, 1973.

MERLEAU-PONTY, Maurice, Signes, Paris, Gallimard, 1960.

PELT, Jean-Marie, Le tour du monde d'un écologiste, Paris, Arthème Fayard, 1990.

ROBINSON, Joan, The Cultural Revolution in China, Harmondsworth, Penguin Books, 1969.

Fin du texte. 